\title{
Estratificación socioeconómica del uso de los prefijos re- y super- en los jóvenes de Bogotá: acercamiento a un estudio diacrónico
}

\section{The socioeconomic stratification of the uses of the prefixes re- and super- in young people's speeches of Bogotá: Towards a diachronic study}

\author{
John Jairo Aguirre Londoño' \\ Juliana Angélica Molina Ríos ${ }^{2}$ \\ Bibiana Yaneth Romero Chala ${ }^{3}$
}

Citation / Para citar este artículo: Aguirre, J. J., Molina, J.A. E Romero, B. Y. (2015). Estratificación socioeconómica del uso de los prefijos re- y super- en los jóvenes de Bogotá: acercamiento a un estudio diacrónico. Colomb. Appl. Linguist. J., 17(1), pp. 42-52.

Received: 10-Apr-2014 / Accepted: 11-Aug-2015

DOI: http://dx.doi.org/10.14483/udistrital.jour.calj.2015.1.a03

\section{Resumen}

En el discurso de los jóvenes es usual la reiteración de algunas expresiones idiomáticas que hacen parte de su identidad y de su pertenencia a un grupo en particular; dicho fenómeno no es ajeno a los jóvenes de la ciudad de Bogotá, en quienes se ha logrado identificar el uso constante de los morfemas apreciativos re- y super-. Este artículo presenta una comparación diacrónica entre los datos hallados en los años 2007 y 2013; se tiene en cuenta la frecuencia de uso de los morfemas re-y super-por parte de los jóvenes bogotanos dentro de la variable social de estratificación socioeconómica. Los resultados generales muestran diferentes tendencias en las tres clases sociales tomadas en cuenta. En la clase baja (estratos 1y 2) se dio una disminución notoria del uso del prefijo re- en el año 2013 en comparación con el 2007; la clase media (estratos 3 y 4), presentó un aumento significativo en el uso del morfema super- en el 2013; y, en la clase alta (estratos 5 y 6) en 2013 disminuyó el uso del prefijo re-, pero incrementó significativamente el uso del prefijo super-. En conclusión, se hace evidente que los jóvenes bogotanos siguen utilizando los prefijos nombrados, aunque con variaciones de uso según la estratificación social.

Palabras clave: afijos apreciativos, estratificación social, morfología, sociolingüística

\begin{abstract}
In young people's speech, the reiteration of certain idiomatic expressions as constitutive of their identity and membership in a particular group is a common occurrence. This phenomenon is not distant to young people in Bogotá, in which they constantly use appreciative morphemes such as re- and super-.
\end{abstract}

This article presents part of the results derived from a research conducted in 2007 concerning this phenomenon.

1 Pontificia Universidad Javeriana, Bogotá D.C., Colombia. aguirre.john@javeriana.edu.co

2 Pontificia Universidad Javeriana, Bogotá D.C., Colombia. juliana.molina@javeriana.edu.co

3 Universidad Pontificia Bolivariana, Medellín, Colombia. bibiromeroch@hotmail.com 
However, six years later, data was collected again by means of the same instrument employed in 2007. Therefore, this article presents a diachronic comparison between the data found in 2007 and 2013 in which both the frequency in use of these morphemes (re- and super-) and the social variable of socioeconomic stratification are considered.

The general results show different trends in the three different socioeconomic levels. In the low levels, in 1 and 2 , as established in Colombia, there was a significant increase in the use of the prefix re- in the year 2013 in comparison to 2007; in the middle levels, 3 and 4, also presented an important increase in the use of the morpheme super- in 2013; and, in the high levels, 5 and 6, in 2013, there was a decrease of the use of the prefix re-, but the use of the prefix superincreased meaningfully. In conclusion, it is evident that young people in Bogota continue using the above mentioned prefixes; although, there are variations in their use according to the socioeconomic level they belong to.

Keywords: morphology, prefixes denoting higher degree, social estratification, sociolinguistics

\section{Introducción}

El uso de diferentes prefijos apreciativos es una característica lingüística de tipo morfológico, muy normal en el habla de los jóvenes; su utilización y alternancia puede señalar fenómenos de identidad y diferenciación generacional, así como mecanismos de creación e innovación lingüística. Por ello, sin lugar a dudas, es este un tema interesante y más tratándose de un estudio que pretende comparar los resultados obtenidos entre dos grupos de jóvenes bogotanos (con siete años de diferencia en la toma de las muestras), a fin de descubrir cuáles son las constantes y diferencias que se presentan en la frecuencia de uso de los prefijos que nos interesan.

En el año 2007 se realizó una investigación preliminar sobre el empleo de los prefijos re- y super- entre los jóvenes bogotanos. Los resultados permitieron establecer que su uso y frecuencia se encuentran sujetos a factores o variables como la clase social, el género y la edad. Algunos de los hallazgos relevantes de ese trabajo fueron los siguientes: a) Existe una utilización frecuente entre los jóvenes bogotanos de los prefijos re- y super- en sus charlas espontáneas o habla coloquial. b) Hay un marcado uso de los dos prefijos por parte de la clase socioeconómica alta (estratos cinco y seis), por encima de las otras dos clases socioeconómicas de la ciudad, (la media y la baja). c) Existe mayor apropiación del prefijo re- entre todos los jóvenes entrevistados. d) Se da un mayor uso del prefijo super- entre los hombres jóvenes de la clase socioeconómica alta. e) En contraste con el anterior punto, se da un mayor uso del re- en la clase baja. $\mathrm{Y}, \mathrm{f})$ hay existencia de un uso preferencial de los prefijos para acompañar adjetivos calificativos y, en segundo lugar, sustantivos.

Años después de la publicación de estos resultados la inquietud por el comportamiento de este fenómeno lingüístico siguió latente. El contacto con estudiantes de diferentes instituciones universitarias (públicas y privadas) de Bogotá, provocó desde las aulas de clase ampliar el interrogante sobre el crecimiento o desaparición de los prefijos apreciativos estudiados, en las charlas cotidianas más elaboradas de los jóvenes e incluso como parte de sus registros más elaborados. Por eso se retoma el instrumento de recolección de la muestra y se decide aplicarlo nuevamente en otro grupo de 60 jóvenes con el propósito de identificar si hubo o no cambios en el uso de los prefijos en mención.

Este artículo se inscribe como un estudio diacrónico en el que se hace énfasis únicamente en la variable dependiente de estratificación social. Mientras tanto la variable lingüística sigue concentrada en el uso de los prefijos re- y superentre los jóvenes bogotanos.

Al revisar la literatura relacionada con la temática en desarrollo, se encuentra que los prefijos del español ha sido un tema de interés entre muchos gramáticos. Es así como Bernardo Morales Ascencio en su artículo "Una caracterización semántica de los prefijos del español" (1995), plantea una tipología de estos morfemas en 
función de la cual es posible caracterizarlos desde su significado. Morales es preciso en afirmar que los "prefijos se caracterizan especialmente por su vaguedad semántica, ligada a la naturaleza misma de los significados expresados" (1995, p. 32). Por eso no es tan difícil entender el aprovechamiento de estos morfemas (y más si son apreciativos), en los discursos casuales de los jóvenes, pues con ello se creería, entre otras cosas, que el discurso evita una amplia responsabilidad significativa.

Por otra parte, tomando como punto de referencia el comportamiento de los prefijos apreciativos, el trabajo de María Isabel Rodríguez Ponce "Los prefijos apreciativos como formantes de plasticwords" (2002) muestra cómo algunos morfemas, afijos, prefijos apreciativos, sirven para construir precisamente, palabras plásticas o palabras ameba (tal y como lo traduce la autora) $y$, que entre otras cosas se caracterizan por ser estereotipos generados a partir de términos científicos, para reemplazar términos tradicionales y producir más allá de su contenido una fonética rimbombante que busca ante todo dar cierto prestigio a las locuciones (2002, p. 2).

Del amplio listado de prefijos apreciativos que sirven como constructores de palabras plásticas, se dedica una importante sesión al prefijo super-. La autora señala que es muy productivo en la construcción de neologías, especialmente en ámbitos como la publicidad y el periodismo. Asimismo, indica que su contenido puede llegar a ser indefinible. En suma, este trabajo muestra el papel fundamental que cumplen prefijos, como super-, para permitir la construcción de dicho tipo de palabras.

Otra referencia importante es la de Iraima Palencia, denominada "La prefijación en el español hablado en Maracaibo" (2005). Esta investigación tiene entre otros objetivos, el de ayudar en la delineación del perfil dialectal de dicha región venezolana. Se interesa por inventariar los prefijos más usados allí y hace una comparación con el mismo fenómeno presentado en la ciudad de Caracas. Al final, se establece que este fenómeno morfológico de derivación se encuentra más acentuado en Maracaibo.
Aunque el trabajo es de corte cuantitativo y sus conclusiones están sujetas a lo que indican los porcentajes, vale la pena mencionar que se hace una contextualización de los prefijos más empleados en Maracaibo y entre ellos aparece uno de los que nos interesa en el presente artículo: el prefijo re-. No obstante dentro de su caracterización, Palencia no encuentra una referencia apreciativa del prefijo. Todas las mencionadas son de tipo significativo.

El lenguaje de los jóvenes es un interés que han tenido muchos investigadores. Gemma Herrero (Rodríguez, 2002) manifiesta que el lenguaje juvenil no puede ser homogenizado o ser visto con una lente reduccionista puesto que es una variedad social de lengua, un sociolecto en el que se ha dado prominencia al factor edad, sobre otros posibles factores sociales de variación: sexo, nivel cultural, clase social y grupos específicos. A raíz de lo anterior, es fundamental considerar que el lenguaje de los jóvenes no puede menos que ser heterogéneo por naturaleza y que factores sociales como el sociocultural, tal y como lo plantea López, permiten demostrar la elocuencia en dichos lenguajes (1983, p. 101).

\section{Referentes teóricos}

Según la Nueva gramática de la lengua española (NGLE), publicada en 2009, la derivación como fenómeno morfológico, debe entenderse en la lingüística contemporánea desde dos perspectivas: primero como oposición a la flexión y a lo que se conoce como morfología flexiva, es decir, aquella que se concentra en la valoración gramatical de la palabra a partir de morfemas que ofrecen información desinencial en la palabra. Es apropiado anotar que en la derivación también caben los fenómenos de composición y parasíntesis y que en un sentido más taxativo, se circunscribe a los procedimientos de formación de palabras a partir de la utilización de afijos simples como prefijos, sufijos o interfijos (NGLE, 2009, p. 337).

Desde la segunda perspectiva de derivación, nos concentramos ahora en la prefijación que es definida por la NGLE como el proceso morfológico por el que se agrega un morfema llamado prefijo a una palabra ya formada como en des-hecho o a 
un tema latino o griego como en a-morfo (2009, p. 663). Resulta importante entender que la ubicación del morfema es fundamental para no confundir la prefijación con la sufijación, y como lo manifiesta Soledad Varela en su Morfología Léxica (2005), para comprender que los prefijos no cambian la categoría gramatical de la base léxica a la que se aplican, sino que se limitan a añadir precisiones al significado del lexema al que preceden.

Interesa ahora determinar la o las clasificaciones a las que pertenecen los dos prefijos que se estudian en este trabajo. Por un lado, Varela (2005) considera que así como re- puede cumplir funciones de posición (detrás) como en rebótica; repetición de la acción como en redecorar; nuevo o segundo como en rellamada; e intensificación de la cualidad como en reguapo. Por su parte, el prefijo super- puede cumplir las funciones de posición (encima de) como en superponer; intensificación del tamaño como en supermercado y exceso e intensificación de la acción o de la cualidad como en superinteresante. Así pues, una de las funciones en común que tienen los dos prefijos (y que aquí nos proponemos identificar) es la de la intensificación.

Por otro lado, el gramático Manuel Seco en su Gramática Esencial de la Lengua Española (GELE) (1999) considera que tanto los sufijos como los prefijos pueden hacer parte de dos amplios grupos: los significativos y los apreciativos. Puede ocurrir que en distintos casos un mismo prefijo actúe de una u otra manera (1999, p. 321). Esto es precisamente lo que sucede con re- y super-. El primero destaca la marca de repetición, y el segundo de superioridad o exceso, esto dentro de la clasificación de grupo significativo. De la misma forma, los dos morfemas se hallan dentro de la clasificación de prefijos apreciativos, al lado de archi, extra y requete.

Interesa también revisar la clasificación de los prefijos propuesta por la gramática (NGLE) a partir de cuatro criterios básicos:

A. La clase de palabras a la que se asimilan.

B. Su dependencia o independencia formal.

C. Su significado.

D. Su relación con las propiedades sintácticas de los predicados.
Si se atiende al primer criterio, los prefijos pueden ser adjetivales como en neogótico o minibar, adverbiales como en entreabrir o prefigurar o preposicionales como en prenatal o subcutáneo. Se entiende que la función cumplida por cada prefijo en las tres clasificaciones, está literalmente relacionada con la función gramatical que se describe en principio; sin embargo, pueden existir ambivalencias generadas por la posibilidad de pertenecer a dos de las clases mencionadas (NGLE, 2009, p. 669). Dentro de esta clasificación, los prefijos re- y superpueden pertenecer a las dos primeras categorías pero es más reiterada su pertenencia a la segunda. $\mathrm{Al}$ respecto algunos ejemplos de los resultados que se obtuvieron, muestran palabras como: superplan, en donde se cumpliría con la primera función, es decir, la adjetival, porque está acompañando a un sustantivo, aunque como lo plantea Varela (2005), no haya una modificación significativa de este. En la palabra remal se cumpliría con la función adverbial si entendemos que los adverbios modifican verbos, adjetivos y adverbios. Por último, vale aclarar que para cumplir con la tercera función, se estaría entrando en el terreno de los prefijos significativos, en el que no se pretende hacer ninguna exploración.

El segundo criterio identifica los prefijos como inseparables en ejemplos del tipo: desproporcionado y separables como en ex marido. Se debe aclara que según la NGLE (2009, p. 669), a los prefijos separables, que también denomina autónomos, exentos o no ligados, el concepto de separabilidad es una característica que solo se debe ver desde una perspectiva morfológica; esto permite que sea relativamente independiente de las convenciones ortográficas del español. Por eso, desde el punto de vista ortográfico, solo hasta el año 2010, se entendía en esta separación, que el prefijo ex (sin guion) cumplía la función de adjetivo al igual que buen o mal. Hoy en día, un ex- (con guion) solo irá antes de una cifra. Lo anterior es fundamental para entender que los prefijos separables en realidad se emplean integrados a la voz a la que anteceden (NGLE 2009).

El tercer criterio obedece a una clasificación semántica, es decir de acuerdo con su significado. La Gramática plantea la siguiente gama: espaciales, temporales, cuantificativos, gradativos y escalares, 
aspectuales, negativos y de orientación o disposición. Nos importa reconocer que los prefijos trabajados en esta investigación se sitúan dentro de los llamados gradativos y escalares de intensidad. No serán entonces espaciales de superioridad como en superponer, ni de posición trasera como en rebotica, de movimiento hacia atrás como en refluir, ni tampoco gradativos escalares de exceso como en superpoblación, ni aspectuales de reiteración como en reintentar.

El último criterio que es el más complejo de los cuatro, se concentra en la morfosintaxis y tiene una gran incidencia en la semántica. Se caracteriza por escudriñar la incidencia argumental de los prefijos en las construcciones oracionales, campo que en el presente artículo no exploraremos.

Con respecto al tercer criterio, el relacionado con el significado de los afijos prefijos apreciativos, (según Seco (1999), o de intensidad como los describe Martín (1998), existe una preocupación por hacer una caracterización morfo-semántica de estos morfemas. En una de las aclaraciones fundamentales de la autora, dice que las categorías gramaticales pueden admitir la intensificación por procedimientos morfológicos —como el que nos interesa en esta investigación - o por procedimientos sintácticos, apelando para esto a la repetición o al adverbio. Se señala entonces que únicamente las categorías léxicas son susceptibles de ser intensificadas: nombres, verbos, adjetivos y adverbios, y se descartan las categorías gramaticales funcionales como la preposición, la conjunción, los pronombres o los artículos (1998, p. 104). En esta categorización es recomendable remitirse a Yule (2007, p. 77), quien plantea la existencia de morfemas léxicos y morfemas funcionales. De los primeros dice que son los portadores del contenido de los mensajes y constituyen una clase abierta de palabras. En cambio los morfemas funcionales o gramaticales, como los artículos y las preposiciones, son una clase cerrada de palabras ya que la incorporación de nuevos morfemas con estas características es muy escasa.

Otro aspecto fundamental para revisar a la luz de Martín (1998) es el relacionado con la clase de adjetivos que admiten el acompañamiento del prefijo intensivo. Es evidente que solo los calificativos pueden ser intensificados, lo que descarta automáticamente a los relacionales (1998, p. 105). Para aclarar esta diferencia, la Gramática nos da ejemplos de adjetivos relacionales como: deportivo y ministerial y calificativos como fabulosa o bonita. Hay una amplia serie de tácticas para diferenciar estas dos clases de adjetivos, de las cuales solo mencionaremos una: mientras el calificativo puede estar antes o después del sustantivo como en fabulosa nave o nave fabulosa, el relacional espacial no puede hacer lo propio con el mismo sustantivo (NGLE, 2011, p. 72).

En cuanto a los sustantivos que pueden ser intensificados, Martín (1998, p. 106) nos dice que los nombres serán intensificados siempre que denoten cualidades o características susceptibles de ser graduadas, lo que explica que algunos de ellos suelan recategorizarse como adjetivos calificativos. Es por eso que las oraciones atributivas permiten que sus núcleos del predicado sean también sustantivos como en el caso de Manuel es taxista.

Otro texto de Soledad Varela y Josefa Martín publicado por Bosque y Monte, en la Gramática Descriptiva de la Lengua Española (GDLE) (2000), nos permite ampliar más la perspectiva de estos fenómenos que hemos mencionado. Las maestras señalan, por ejemplo, que los prefijos pueden tener un origen con valor adverbial ya bien sea porque provienen de un adverbio (no- o mal-), de una preposición española (sobre-, entre- o super-) o griega (hipercrítico) o de prefijos latinos (re-, semi-) (2000, p. 5001). Dicen que además de los adverbios, estos prefijos modifican predicados, al adjuntarse a bases verbales y a bases adjetivas para afectar la acción o situación expresada en el verbo base $\mathrm{o}$ incluso la propiedad denotada por el adjetivo (2000, p. 5001).

Las autoras señalan también que los prefijos adverbiales despliegan distintas nociones semánticas y un mismo prefijo, incluso puede presentar más de un contenido semántico (2000, p. 5001). Lo anterior lo podemos ejemplificar con los prefijos re- y super- que estamos estudiando, pues re- tiene valor de posición, intensidad de la cualidad e iteración. Por su parte, super- tiene 
valores como: posición, intensidad de la cualidad e intensidad del tamaño. Las autoras también sostienen que el prefijo super- es el más productivo de todos los prefijos en el español tanto de América como de España porque puede unirse a nombres (supertarjeta), adjetivos (superrígido) o verbos (superpoblar). Además, puede funcionar en forma libre en contextos como: gasolina súper (2000, p. 5027). Con esto podemos recrear la asimetría existente entre los dos prefijos pero también ver la coincidencia en el valor de intensidad de la cualidad, noción que como veremos es la que más se presenta en este trabajo.

Por último, si se hace un rastreo de la génesis de los prefijos re- y super-, es fácil advertir en este último, un aspecto tan determinante como el nacimiento de la industria del cine y el desarrollo de la aviación. En una investigación realizada por Oroz, intitulada Algunos rasgos característicos del vocabulario contemporáneo chileno (1969), el autor señala que se pueden presentar dos combinaciones muy comunes entre, por un lado, el prefijo super- más un sustantivo; y por el otro lado, el prefijo más un adjetivo. En el primero, existen ejemplos como el de superbomba o supersalón y en el segundo, ejemplos con adjetivos relacionales como superenamorado o superpoblado. Oroz (1969) ya había realizado algunos trabajos sobre el uso de estos prefijos en el país austral, y anotaba además que el prefijo super- a pesar de ser bastante antiguo y de tener una línea de evolución similar en casi todas las lenguas romances, ya que pasó del valor local relacionado con la extensión o límite superficial al valor de intensidad, tomó una fuerza descomunal en el siglo XX con el desarrollo de la aviación y la cinematografía. De allí nacieron entonces, vocablos como superbombardero, superfortaleza y superproducción, por solo citar algunos.

De este modo, se puede advertir que los prefijos apreciativos o intensivos, han cumplido un papel que como lo manifiesta María Isabel Rodríguez (2002), permite construir palabras que dejan en un segundo plano el aspecto significativo y le apuestan más a un tono prestigioso, ampliamente connotativo, que reemplaza las voces obsoletas que se revisten de términos tradicionales. Por eso son prefijos apreciativos, porque simplemente se conforman con apreciar, desde una perspectiva subjetiva, por medio de la palabra, y son intensivos porque la sonoridad y la rimbombancia se superponen al contenido mismo. Desde luego, esto termina siendo muy propio del registro coloquial o incluso vulgar y un fenómeno más desarrollado en el español de América que en el peninsular según Soledad Varela y Josefa Martín (2000, p. 5026).

\section{Metodología}

El presente ejercicio sociolingüístico se puede establecer como un estudio diacrónico, puesto que el objetivo es comparar la frecuencia de uso de un fenómeno lingüístico en dos momentos precisos de la historia del español hablado por los jóvenes de Bogotá: años 2007 y 2013, teniendo en cuenta para ello la variable de estratificación social.

En este sentido, se empleó la estadística descriptiva con el fin de cuantificar y describir el comportamiento de los datos obtenidos en 2007 y 2013. Según Moreno (1990, p. 123), el uso de la estadística en el estudio de la lengua y la sociedad cumple dos reglas: a) describir y resumir los datos; y b) hacer estimaciones de significación y fiabilidad. A su vez, se recurrió a la frecuencia; en términos de Moreno (1990, p. 127), las frecuencias suelen presentarse de dos formas: bien como frecuencias absolutas (el número real de veces que aparece un elemento), bien como frecuencias relativas, llamadas también proporciones o porcentajes (la proporción de casos respecto de un total en que aparece un elemento); así, en este ejercicio diacrónico se tuvieron en cuenta los dos tipos de frecuencias antes mencionados.

La población que hizo parte de esta investigación está constituida por jóvenes que estuvieran entre los 15 y los 32 años de edad. Para fijar este criterio de juventud, recurrimos a Félix Rodríguez (2002) quien anota que la mayoría de los estudios sociológicos, sobre todo si son de carácter empírico, sitúan la juventud entre los quince y los veinticuatro años de edad tal y como lo define la ONU. Sin embargo, otros creen que se debe entender el ocaso de la juventud, no tanto por el termómetro biológico o cronológico sino por la reunión de algunas características de 
la personalidad que afloran antes o después de lo comúnmente previsto:

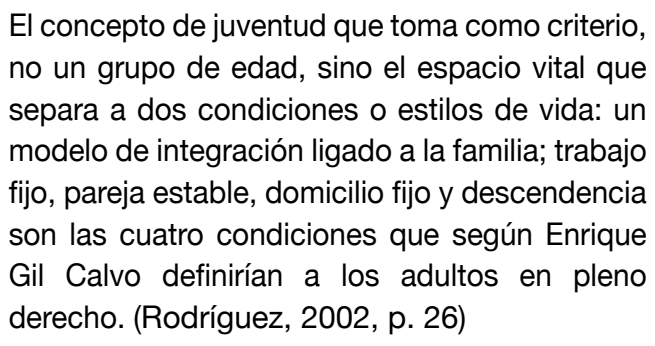

El concepto de juventud que toma como criterio, no un grupo de edad, sino el espacio vital que separa a dos condiciones o estilos de vida: un modelo de integración ligado a la familia; trabajo fijo, pareja estable, domicilio fijo y descendencia son las cuatro condiciones que según Enrique Gil Calvo definirían a los adultos en pleno derecho. (Rodríguez, 2002, p. 26)

Para la sociolingüística las poblaciones que se manejan están estratificadas de acuerdo con variables lingüísticas y sociales. En este caso, el elemento lingüístico se relaciona con el nivel morfológico de la lengua española, específicamente con el uso de los morfemas apreciativos re- y super-, y el social con la estratificación socioeconómica. En la ciudad de Bogotá, la estratificación social está distribuida en tres niveles: estrato bajo (uno y dos), estrato medio (tres y cuatro) y estrato alto (cinco y seis). Asimismo, se tuvo en cuenta que los informantes fueran oriundos de Bogotá o que llevaran más de diez años viviendo en esta ciudad.

A continuación se relaciona el número de la población, tanto de 2007 como de 2013, que conformó la muestra total (el universo total a analizar):

Tabla 1.

\begin{tabular}{cccc}
\hline & 2007 & & \multicolumn{2}{c}{$\mathbf{2 0 1 3}$} \\
\hline Estrato alto & 20 jóvenes & Estrato alto & 20 jóvenes \\
Estrato medio & 20 jóvenes & Estrato medio & 20 jóvenes \\
Estrato bajo & 20 jóvenes & Estrato bajo & 20 jóvenes \\
Total & 60 jóvenes & Total & 60 jóvenes \\
& Total de la población: 120 jóvenes & \\
\hline
\end{tabular}

Fuente: elaboración propia.

La recolección de datos realizada en 2007 se realizó mediante la aplicación de un cuestionario organizado en dos partes. En la primera parte se pedía al informante que respondiera unas preguntas relacionadas con las impresiones generadas por una serie de fotografías, la mayoría de las cuales eran de personajes de la actualidad política (el expresidente norteamericano George Bush), el deporte (el exfutbolista argentino Batistuta), la música (Juanes y Shakira) o la farándula (la modelo Claudia Bahamón). Además aparecían fotografías de algunos paisajes (bosque y playa), la de un perro hush puppy, la de un bebé obeso, un plato de fresas con crema, un automóvil lujoso y finalmente la de un retrete (objeto de broma) lleno de materia fecal y la de un cadáver descuartizado sobre una mesa.

La segunda parte del cuestionario presentaba 16 preguntas que de igual manera buscaban indagar sobre las impresiones suscitadas por las siguientes situaciones hipotéticas: un celular que se solo se activa con nuestro timbre de voz, una puntilla en un tobogán, una beca para estudiar cualquier carrera en el país que escogiera y con la posibilidad de ejercer allí mismo al terminar, una espera obligada de tres horas en una fila que no se mueve, una cerveza bien fría a la orilla del mar Caribe y bajo una temperatura de 29 grados centígrados, la aparición de una araña negra del tamaño de una mano y justo en el momento de despertar, alguien que se endeuda para prestarte el dinero pero que no se lo dice en ningún momento, el aliento a cebolla en una persona que le atrae, un amigo que le canta el cumpleaños a todo pulmón en el bus e invita a los demás pasajeros a que lo acompañen, el olor a pescado, su plato favorito después de dos días de ayuno obligado, la violencia de nuestro país, el rocío de la mañana, viajar en Transmilenio, oír la canción favorita justo después de dos meses de estar en la India y una enfermedad incurable. 
El mismo cuestionario, sin ninguna modificación, se realizó en el año 2013, pero en este momento algunas imágenes resultaron anacrónicas; por ejemplo, las relacionadas con personajes como el ex presidente norteamericano George Bush y el ex futbolista argentino Gabriel Batistuta. Sin embargo, contextualizamos a los informantes sobre dichos personajes. La intención de aplicar el mismo cuestionario en 2013 era hacer lo más fiel posible la recolección de datos a la forma como se realizó en 2007, puesto que no queríamos que ningún elemento nuevo se convirtiera en foco de desviación.

En este punto es importante aclarar que los cuestionarios que se aplicaron en 2007 superan los 120 informantes (uno por cada informante), mientras los aplicados en 2013 fueron 60. En este sentido, y para que la muestra total estuviera equilibrada, de la muestra de 2007 se retomaron 60 cuestionarios aleatoriamente. Así, se logró la recolección de la muestra total de los datos para la posterior descripción y comparación de frecuencias de uso de los años 2007 y 2013.

\section{Análisis cuantitativo}

Después de realizar la tabulación de los datos obtenidos con la muestra que se levantó en el año 2007 (de la cual tomamos aleatoriamente 60 cuestionarios) y en el 2013, se compararon dichos resultados y se encontraron datos interesantes en el comportamiento de la muestra total, los cuales se presentarán a continuación.

\section{Muestra total}

De las 60 entrevistas realizadas en el 2007 hubo un uso total de los dos prefijos re- y super- en 91 ocasiones en las respuestas de 37 informantes. Esto nos da un promedio de uso de los morfemas, entre el total de la muestra, del $1.5 \%$ y por cuestionarios realizados del $2.45 \%$.

Seis años después, es decir en el 2013, de los 60 cuestionarios aplicados, 43 personas usaron por lo menos una vez alguno de los dos prefijos, con un total de 108 respuestas en las que involucraban su utilización, lo que nos da un promedio de uso entre el total de la muestra del $1.8 \%$ y por cuestionarios realizados del $2.51 \%$. Notamos con esto que el uso en su totalidad de los dos morfemas aumentó un poco en el vocabulario de los jóvenes bogotanos en un $0.3 \%$, si comparamos el total de las dos muestras, y en un $0.06 \%$ si comparamos entre hablantes que hicieron un uso efectivo de alguno de los morfemas, es decir por cuestionario. Los resultados también nos permiten comprender que en el año 2007, el 38.4\% de los entrevistados no hizo uso de los prefijos mientras que en el 2013 ese promedio disminuyó, pues solo el $28.3 \%$ de los entrevistados obvió el uso de dichos morfemas.

Discriminando mucho más los resultados obtenidos durante el 2007, se entiende que hubo un total de 91 ocasiones de uso entre los 37 (de los 60) encuestados, de los prefijos en estudio. Esa cifra se distribuyó de la siguiente manera: en la clase alta se usaron en 37 oportunidades, lo cual representa el $40.6 \%$; en la clase media se empelaron en 20 oportunidades para un $21.9 \%$ y en la clase socioeconómica baja se utilizaron en 34 oportunidades para consolidar un $37.3 \%$.

Los resultados, obtenidos seis años más tarde y sobre la base de 108 como número de uso total entre 43 (de los 60) entrevistados, se comportaron de la siguiente manera: en la clase alta se usaron en 45 oportunidades correspondientes al $41.6 \%$ de la muestra; en la clase media se utilizaron en 39 oportunidades, lo cual constituye el $36.1 \%$ y en la clase baja hubo respuestas con los prefijos estudiados en 24 oportunidades equivalente al $22.2 \%$. Como vemos, a pesar de que los porcentajes no coinciden, sí se presenta un leve aumento en el uso de los prefijos, primero en la clase alta, y un aumento significativo en la clase media; por último, en la clase baja la disminución del uso de los dos prefijos fue notoria.

Las diferencias entre los resultados obtenidos en la clase alta, equivalen al $1 \%$, porcentaje que corresponde al aumento en el uso de los prefijos desde el año 2007 hasta el 2013. La diferencia que se presenta entre las muestras de la clase media es del $14.2 \%$, y también obedece a un aumento significativo desde el año 2007 hasta el año 2013. 
Todo lo contrario ocurrió en la comparación de las dos muestras de la clase socioeconómica baja. La diferencia que existe entre una y otra muestra es del $15.1 \%$, pero este porcentaje representa la disminución en la utilización del morfema en las respuestas de los jóvenes de clase socioeconómica baja.

\section{Uso del prefijo re-}

En cuanto al uso del prefijo re- por clase socioeconómica, se puede anotar que en la investigación del año 2007, este morfema se usó en un total de 64 veces y la clase socioeconómica que más lo utilizó en las respuestas fue la baja, con un total de 28 oportunidades y un porcentaje del $43.7 \%$. En el 2013, el número de veces que se utilizó fue dieciocho para consolidar el $72 \%$ sobre el número de respuestas efectivas. En segundo lugar, se ubicó la clase socioeconómica alta con un uso del prefijo en veinte respuestas, lo que equivale al $31.25 \%$. En el 2013, la clase alta volvió a situarse en el segundo lugar con un uso de cinco veces y un porcentaje del $20 \%$. Finalmente, el último lugar lo ocupó en la investigación del 2007 la clase media. En esta clase se usó el morfema re- en dieciséis oportunidades para consolidar el $25 \%$, mientras que en el 2013 se usó solo en dos oportunidades para marcar un porcentaje del $8 \%$. Así, se observa que el uso del prefijo disminuyó notoriamente, pero se mantuvieron en los mismos lugares las tres clases socioeconómicas. Sin embargo, la clase baja incrementó la utilización del morfema re-, pues pasó del $43.7 \%$ en el 2007 al $72 \%$ en el 2013. En la clase media, del $25 \%$ en el 2007 se redujo al $8 \%$ en el 2013 y en la clase alta, se pasó del $31.2 \%$ al $20 \%$ en el 2013.

\section{Uso del prefijo super-}

En relación con el uso del prefijo super-, se puede señalar que los resultados obtenidos en el año 2013 mostraron algunos cambios interesantes con respecto al 2007 y también se presentaron algunas repeticiones. La clase alta en el 2007 usó el prefijo super- en 17 oportunidades, es decir, el $62.9 \%$, mientras que en el 2013 lo usó 40 veces para consolidar el $48.19 \%$. Vemos como caso interesante que aunque el número de veces usado del prefijo aumentó significativamente entre los dos años, el porcentaje de uso disminuyó en un $14.8 \%$, debido al total de la muestra que implicó un aumento en el uso por parte de las otras dos clases socioeconómicas. El segundo lugar lo ocupó en el 2007 la clase socioeconómica baja con un uso del prefijo en seis ocasiones, es decir el $22.2 \%$ y este mismo número de seis ocasiones se repitió en el 2013 aunque el porcentaje cambió pues fue de tan solo el 7.2\%. Por último, en el año 2007 la clase media ocupó el último lugar en cuanto al uso del morfema super-, con solo cuatro respuestas que incluyeron su utilización. Pero el dato que sorprende es el que nos enseña el 2013 pues de 4 pasó a 37 oportunidades en las que se usó este prefijo; en porcentajes, se pasó del $14.8 \%$ al $44.5 \%$. En resumen, la clase socioeconómica alta se mantuvo como la que más utilizó el afijo prefijo super-, seguida muy de cerca por la clase media.

\section{Análisis cualitativo: aproximaciones}

Además de los datos expuestos, los cuestionarios permitieron detectar las categorías gramaticales que más se presentan en el lenguaje de los jóvenes para ser acompañadas por los prefijos re- y super-. Encontramos, como era de esperarse, en la mayoría de los casos un uso de adjetivos (relinda, superdelicioso), en segundo lugar el uso de la expresión super- sin adherirse a ninguna palabra y de allí en adelante, con ocasiones muy contadas, descubrimos la posposición de algunos adverbios (supercerca), algunos sustantivos (superpersonaje, superplan) o sustantivos adjetivados (repaila).

\section{Connotaciones positivas y negativas de las palabras acompañadas por los prefijos re- $y$ super- en las dos muestras}

En el año2007, las palabras que más se utilizaron (acompañadas de uno de los prefijos estudiados) y con una connotación positiva fueron en su orden de mayor a menor uso: rebueno(a). En segundo lugar apareció el uso de la palabra super- (sin ningún tipo de acompañamiento). Otros usos positivos pero con menor frecuencia en las respuestas fueron rebien (como adverbio), rebuenísimo (adjetivo superlativo) y rebonito, rebacano, superbonito, supertierno y 
supergordo (como adjetivos). En el año 2013, las que se utilizaron en más de una ocasión por uno o diferentes hablantes y con un sentido positivo fueron: super- (sin ningún acompañamiento), rerrico (a), rechimba, remaquia (de la cual no fue claro su significado) y relinda. Es necesario hacer claridad en cuanto al hecho de que cuando se usa únicamente la palabra super-, se podría pensar que se trata de un adjetivo y no de un prefijo apreciativo; sin embargo, en las acepciones que tiene el DRAE, la primera que explica esta palabra como adjetivo dice: "Dicho de la gasolina: De octanaje superior al considerado normal", que discrepa completamente con la que usaron los jóvenes en su respuesta. Creeríamos que se debe a un uso apocopado de la que originalmente pudo haber sido la expresión que desearon decir, un caso que merece un estudio particular.

En cuanto al sentido negativo, la palabra que más se usó en la muestra del 2007 fue remal. En el año 2013, la forma negativa más usada fue refeo (en dos ocasiones).

\section{Otros usos de los prefijos en las muestras}

Otros palabras que fueron usadas en alguna ocasión en las dos muestras, que pueden dar una idea de la amplitud del fenómeno morfológico y que son usadas para calificar positivamente fueron: supertierno, rebalsiado, superlinda, repilas, reducho, reloca, superamigo, rechurríssimo, superrico, supersencillo, rechevre, refull, relegal, superagradable y resevero. $\mathrm{Y}$, en cuanto a las de connotación negativa, se suman: remamón, remaluco, reafiebrado, repaila, recerdo y rebobo. Así, se concluye que fueron más las expresiones de carácter positivo que las de carácter negativo las que estuvieron acompañadas de los morfemas estudiados.

\section{Reflexiones finales}

El presente estudio nos permite develar algunas reflexiones importantes sobre un fenómeno lingüístico particular entre los jóvenes bogotanos como es el uso de los morfemas, afijos prefijos apreciativos re- y super-. A partir de la comparación cuantitativa realizada entre los resultados del 2007 y los del 2013 después de hacer los dos muestreos, se puede señalar lo siguiente:

El uso de los dos prefijos re- y super- (en conjunto) entre los jóvenes bogotanos es constante y en algunos casos se aprecia su aumento. Esto último se advierte primero en la clase alta, seguida de un crecimiento significativo en la clase media; por último y en contraposición con lo anterior, en la clase baja la disminución del uso de los dos prefijos fue notoria.

En los dos años tomados en cuenta para este estudio diacrónico, se evidencia que la clase socioeconómica alta de Bogotá (estratos cinco y seis), emplea mucho más el prefijo super-, mientras que la clase socioeconómica baja (estratificaciones uno y dos) usa más el prefijo re-; sin embargo, la clase media presentó un aumento significativo en el uso del morfema super- entre el 2007 y el 2013.

En cuanto al uso del prefijo re- entre los jóvenes de las clases media y alta, se evidenció que este disminuyó entre el 2007 y el 2013, y en la clase baja a pesar de mantenerse en el primer lugar durante las dos muestras, también marcó una disminución notoria en su utilización en 2013.

Por otra parte, el análisis cualitativo mostró que la categoría gramatical más usada con los prefijos re- y super- fue el adjetivo, seguida del uso solo del prefijo super-, y por último de algunos pocos adverbios, sustantivos y sustantivos adjetivados.

\section{Referencias}

Herrero G. (2002). Aspectos sintácticos del lenguaje juvenil. En Rodríguez, F. (Ed.). El lenguaje de los jóvenes. (p. 67). Barcelona: Ariel.

López, H. (1983). Estratificación social del español en San Juan de Puerto Rico. México: Universidad Nacional Autónoma de México.

Martín, J. (1998). Los prefijos intensivos del español: caracterización morfo-semántica. E.L.U.A., 12, 103-116 Recuperado de http://rua.ua.es/dspace/ bitstream/10045/6331/1/ELUA_12_07.pdfE.L.U.A., 
Morales, B. (1995). Una caracterización semántica de los prefijos del español. Revista Forma y Función, 8.

Moreno, F. (1990). Metodología sociolingüística. Madrid: Gredos.

Oroz, R. (1969). Algunos rasgos característicos del vocabulario contemporáneo chileno. En Boletín de Filología. Tomo XX. Santiago de Chile: Universidad de Chile.

Palencia, I. (2005). La prefijación en el español hablado en Maracaibo. Letras, 47(71). Recuperado de http://www.scielo.org.ve/scielo.php?pid=S045912832005000200004 Escript $=$ sci arttext

Real Academia Española. (2009). Nueva gramática de la lengua española. Volumen I. Madrid: Espasa Libros.

Rodríguez, F. (2002). El lenguaje de los jóvenes. Barcelona: Ariel.
Rodríguez, M. I. (2002). Los prefijos apreciativos como formantes de plasticwords. Anuario de estudios filológicos, 25, 417-432. Recuperado de http:// dialnet.unirioja.es/servlet/articulo?codigo $=298627$

Seco, M. (1999). Gramática Esencial de la Lengua Española. Madrid: Espasa.

Varela, S. y Martín J. (2000). La Prefijación. En I. Bosque y V. Monte (Eds.). Gramática Descriptiva de la Lengua Española. Tomo 3. Capítulo 76. (pp. 4993- 5040). Madrid: Espasa.

Varela, S. (2005). Morfología Léxica: la formación de palabras. Madrid: Gredos.

Yule, G. (2007). El Lenguaje. Madrid: Akal. 